\title{
Oncolytic H-1 Parvovirus Enters Cancer Cells through Clathrin-Mediated Endocytosis
}

\author{
Tiago Ferreira ${ }^{1}\left(\right.$, Amit Kulkarni ${ }^{2} @$, Clemens Bretscher ${ }^{1}$, Karsten Richter ${ }^{3}$, Marcelo Ehrlich ${ }^{4}(\mathbb{D}$ \\ and Antonio Marchini 1,2,* \\ 1 Laboratory of Oncolytic Virus Immuno-Therapeutics, German Cancer Research Centre, \\ Im Neuenheimer Feld 242, 69120 Heidelberg, Germany; t.ferreira@dkfz.de (T.F.); c.bretscher@dkfz.de (C.B.) \\ 2 Laboratory of Oncolytic Virus Immuno-Therapeutics, Luxembourg Institute of Health, 84 Val Fleuri, \\ L-1526 Luxembourg, Luxembourg; amit.kulkarni@lih.lu \\ 3 Core Facility Electron Microscopy, German Cancer Research Centre, Im Neuenheimer Feld 280, \\ 69120 Heidelberg, Germany; k.richter@dkfz-heidelberg.de \\ 4 Laboratory of Signal Transduction and Membrane Biology, The Shumins School for Biomedicine and Cancer \\ Research, George S. Wise Faculty of Life Sciences, Tel Aviv University, 69978 Tel Aviv, Israel; \\ marceloe@post.tau.ac.il \\ * Correspondence: a.marchini@dkfz.de or antonio.marchini@lih.lu; Tel.: +49-6221-424969 or +352-26-970-856
}

Received: 8 October 2020; Accepted: 19 October 2020; Published: 21 October 2020

\begin{abstract}
H}-1$ protoparvovirus (H-1PV) is a self-propagating virus that is non-pathogenic in humans and has oncolytic and oncosuppressive activities. H-1PV is the first member of the Parvoviridae family to undergo clinical testing as an anticancer agent. Results from clinical trials in patients with glioblastoma or pancreatic carcinoma show that virus treatment is safe, well-tolerated and associated with first signs of efficacy. Characterisation of the H-1PV life cycle may help to improve its efficacy and clinical outcome. In this study, we investigated the entry route of H-1PV in cervical carcinoma HeLa and glioma NCH125 cell lines. Using electron and confocal microscopy, we detected H-1PV particles within clathrin-coated pits and vesicles, providing evidence that the virus uses clathrin-mediated endocytosis for cell entry. In agreement with these results, we found that blocking clathrin-mediated endocytosis using specific inhibitors or small interfering RNA-mediated knockdown of its key regulator, AP2M1, markedly reduced H-1PV entry. By contrast, we found no evidence of viral entry through caveolae-mediated endocytosis. We also show that H-1PV entry is dependent on dynamin, while viral trafficking occurs from early to late endosomes, with acidic $\mathrm{pH}$ necessary for a productive infection. This is the first study that characterises the cell entry pathways of oncolytic H-1PV.
\end{abstract}

Keywords: oncolytic viruses; rodent protoparvovirus H-1PV; virus entry; clathrin-mediated endocytosis

\section{Introduction}

The rodent $\mathrm{H}-1$ protoparvovirus (H-1PV) belongs to the Parvoviridae family, genus Protoparvovirus [1]. This genus also includes Rodent protoparvovirus 1 (H-1PV, Kilham rat virus, LuIII virus, minute virus of mice (MVM), mouse parvovirus, tumour virus $X$, rat minute virus), Rodent protoparvovirus 2 (rat parvovirus 1), Carnivore protoparvovirus 1 (canine parvovirus (CPV) and feline panleukopenia parvovirus (FPV)), Primate protoparvovirus 1 (bufavirus) and Ungulate parvovirus 1 (porcine parvovirus (PPV)) [2,3]. Protoparvoviruses (PtPVs) are single-stranded DNA viruses with an icosahedral capsid of about $25 \mathrm{~nm}$ diameter. Their genomes encompass the non-structural (NS) and the viral particle (VP) transcriptional units, whose expressions are regulated by the P4 and P38 promoters, respectively. The NS transcriptional unit encodes the NS1 and NS2 proteins, whereas the VP transcriptional unit encodes the VP1 and VP2 capsid proteins and the small alternatively translated protein [4]. 
Owing to their ability to specifically infect, replicate and kill human cancer cells, rodent PtPVs are under investigation as potential anticancer therapies. Pre-clinical studies have revealed that H-1PV in particular has remarkable oncolytic and oncosuppressive activity in a number of cell culture and animal models of cancers from different origins [5]. Notably, H-1PV-induced cancer cell death and lysis are immunogenic and stimulate the immune system to participate in the elimination of cancer cells [6]. NS1 is the major effector of H-1PV oncotoxicity [7].

Although viral oncolytic activity is shared between rodent PtPVs, H-1PV is the only member of the genus to have reached the clinic as an anticancer therapy. In a phase I/IIa clinical trial in patients with recurrent glioblastoma (ParvOryx01), H-1PV treatment was safe, well-tolerated and associated with first evidence of anticancer efficacy. This evidence included the ability of H-1PV to cross the blood-brain barrier after intravenous administration, its wide distribution in the tumour bed, the induction of tumour necrosis and immuno-conversion of the tumour microenvironment. As a result, virus treatment led to an improved progression-free survival and median overall survival of patients in comparison with historical controls [8]. A dose-escalation phase I/IIa pilot study in patients with metastatic pancreatic cancer recently confirmed the excellent safety and tolerability of H-1PV treatment. In accordance with the results of ParvOryx01, patients who responded to the treatment showed evident changes in the tumour microenvironment and induction of specific immune responses [9].

The PtPV life cycle is strictly dependent on host cellular factors for a productive infection, from cell surface attachment and entry to virus DNA replication, gene expression, multiplication and egress. Some of these factors are frequently overexpressed or dysregulated in cancer cells. The list includes cell cycle regulators, transcription factors, modulators of the DNA damage response, kinases and cytoskeleton components (reviewed in Reference [10]). However, unlike for other PtPVs, the early steps of $\mathrm{H}-1 \mathrm{PV}$ infection remain to be characterised.

The first interaction between PtPVs and the target cell occurs through binding to a specific surface receptor exposed on the host plasma membrane. Cellular receptors for some PtPVs have been described, such as the transferrin receptor for CPV and FPV. H-1PV, like MVM and PPV, uses sialic acid (SA) for cell surface attachment and entry. However, it is unclear whether SA itself acts as a functional viral receptor for the virus or is a component of an as yet unidentified receptor(s) or receptor complex [3,11,12].

After docking to the cellular membrane, viruses are internalised through different pathways [13]. Clathrin- and caveolae-mediated endocytosis are two dynamin-dependent pathways, whereas macropinocytosis, lipid-raft-mediated endocytosis and caveolae/clathrin-independent endocytosis are dynamin-independent pathways [14,15]. Clathrin-mediated endocytosis is the pathway commonly used by small viruses, including PtPVs [16-20]. The mechanism begins with the recruitment of adaptor protein 2 (AP-2) complexes on the plasma membrane, followed by the assembly of a three-dimensional clathrin coat that leads to a progressive invagination of the membrane. Dynamin self-assembles around the vesicle neck and mediates its scission, and the vesicle is subsequently released into the interior of the cell [21].

PtPVs also use alternative endocytic pathways. For instance, MVM prototype strain takes at least three different endocytic routes: clathrin-, caveolae- and clathrin-independent carrier-mediated endocytosis [22]. Even though endocytosis seems to be the default entry pathway for PtPVs, differences between members of the family may contribute to the tropism of these viruses.

As the PtPV is trafficked within the cellular endosome, its capsid undergoes slow structural changes. In particular, the acidic environment exposes the catalytic phospholipase 2 domain of VP1. This conformational change promotes the digestion of the endosomal membrane, resulting in the release of viral particles from the late endosome to the cytosol [3]. Thereafter, incoming PtPV particles are transported to the nucleus in a process that is dependent on the cytoskeleton and associated motor proteins [23]. 
In this study, we used electron microscopy (EM) and immunofluorescence (IF), together with a number of chemical inhibitors and siRNA-mediated knockdown, to identify which of these pathways $\mathrm{H}-1 \mathrm{PV}$ uses to enter cancer cells. We found that H-1PV cell uptake occurs preferentially through clathrin-mediated, but not caveolae-mediated, endocytosis in cervical carcinoma HeLa and glioma $\mathrm{NCH} 125$ cell lines. Entry was also dependent on dynamin activity. We show that after its internalisation, $\mathrm{H}-1 \mathrm{PV}$, like other PtPVs, passes through early endosomes to late endosomes/lysosomes during its cytosolic trafficking. Productive infection relies heavily on the acidic $\mathrm{pH}$ in the endosomes.

\section{Materials and Methods}

\subsection{Cells and Viruses}

The cervical carcinoma-derived HeLa [7] and the glioblastoma-derived NCH125 cell lines [24] were cultured in Dulbecco's modified Eagle's medium (DMEM) supplemented with 10\% FBS, 100 units/mL penicillin, $100 \mu \mathrm{g} / \mathrm{mL}$ streptomycin and $2 \mathrm{mM}$ L-glutamine (all from Gibco, Thermo Fischer Scientific, Darmstadt, Germany) in a humidified incubator at $37^{\circ} \mathrm{C}$. Both cell lines were tested for mycoplasma contamination by PCR in a regular base.

Both wild-type $\mathrm{H}-1 \mathrm{PV}$ and recombinant $\mathrm{H}-1 \mathrm{PV}$ harbouring the green fluorescent protein-encoding gene (recH-1PV-EGFP) were produced, purified and titrated as previously described $[25,26]$.

\subsection{Electron Microscopy}

HeLa cells were seeded on punched sheets of ACLAR-Fluoropolymer films (Electron Microscopy Sciences) at a density of $8 \times 10^{4}$ cells/well in 24 -well plates. On the following day, cells were infected with $\mathrm{H}-1 \mathrm{PV}$ at a multiplicity of infection (MOI) of 2000 plaque forming units (pfu) per cell in DMEM $5 \%$ FBS at $4{ }^{\circ} \mathrm{C}$ for $1 \mathrm{~h}$ to allow virus attachment to the cell surface and promote a synchronised infection. In order to catch the internalisation event, cells were shifted to $37^{\circ} \mathrm{C}$ for $0,5,10,20$ and $30 \mathrm{~min}$. After incubation, ACLAR-Fluoropolymer films were embedded in epoxy resin for ultrathin sectioning according to standard procedures. Briefly, chemical fixation was carried out in buffered aldehyde ( $4 \%$ formaldehyde, $2 \%$ glutaraldehyde, $1 \mathrm{mM} \mathrm{CaCl}_{2}, 1 \mathrm{mM} \mathrm{MgCl}_{2}$ in $100 \mathrm{mM}$ Ca-cacodylate, pH 7.2), followed by post-fixation in buffered $1 \%$ osmium tetroxide and en bloc staining in $1 \%$ uranylacetate. Following dehydration in graded steps of ethanol, the adherent cells were flat-embedded in epoxy resin (mixture of glycid ether, methylnadic anhydride and dodecenyl-succinic-anhydride; Serva). Ultrathin sections of nominal thickness $60 \mathrm{~nm}$ and contrast-stained with lead-citrate and uranylacetate were analysed using a Zeiss EM 910 (Carl Zeiss, Oberkochen, Germany) at 120 kV and micrographs taken using a slow scan charge-coupled device camera (TRS, Olympus, Moorenweis, Germany).

\subsection{Co-Localisation of H-1PV and Cellular Proteins by Confocal Microscopy}

HeLa cells were seeded at a density of $3.5 \times 10^{3}$ cells/spot on spot slides and grown in $50 \mu \mathrm{L}$ of complete cellular medium. On the following day, cells were placed on ice for $15 \mathrm{~min}$ and then infected with wild-type H-1PV at a MOI of 500 (pfu/cell) in a total of $70 \mu \mathrm{L}$ of $5 \%$ FCS-containing medium. At $1 \mathrm{~h}$ post-infection, cells were shifted to $37^{\circ} \mathrm{C}$ for varied times depending on the experiment, before being fixed with $3.7 \%$ paraformaldehyde on ice for $15 \mathrm{~min}$ and permeabilised with $1 \%$ Triton X-100 for $10 \mathrm{~min}$. Immunostaining was carried out with the following antibodies, all used at dilution 1:500 for $1 \mathrm{~h}$ : mouse monoclonal anti-H-1PV capsid (a conformational antibody kindly provided by Barbara Leuchs; DKFZ Virus Production and Development Unit, Heidelberg, Germany) [27], rabbit monoclonal anti-clathrin heavy chain (D3C6; Cell Signalling Technology, Leiden, Netherlands), rabbit monoclonal anti-EEA1 (3288; Cell Signalling Technology), rabbit monoclonal anti-Rab7 (9367T; Cell Signalling Technology) and rabbit polyclonal anti-LAMP-1 (CD107a) (AB2971; Merck, Darmstadt, Germany). Anti-mouse Alexa Fluor 594 IgG (A11005; Thermo Fisher Scientific, Bleiswijk, Netherlands) or anti-rabbit Alexa Fluor 488 IgG (A11008; Thermo Fisher Scientific) were used as secondary antibodies. Nuclei were stained by 4',6-diamidin-2-phenylindol (DAPI). Images in the green channel (H-1PV), red channel 
(varied cellular proteins) or blue channel (DAPI) were acquired with a confocal microscope (Leica TCS SP5 II, Wetzlar, Germany). Picture analysis was carried out using the Leica LAS X Software.

\subsection{Treatment with Inhibitors of Endocytosis Pathways}

Hypertonic sucrose (Carl Roth), 0.40 M, chlorpromazine (Sigma-Aldrich Chemie GmbH, Steinheim, Germany), $2.5 \mu \mathrm{g} / \mathrm{mL}$ for HeLa or $5 \mu \mathrm{g} / \mathrm{mL}$ for NCH125, and pitstop 2 (Sigma-Aldrich Chemie GmbH), $30 \mu \mathrm{M}$, were used to inhibit clathrin-mediated endocytosis. Dynole ${ }^{\mathrm{TM}}$ Series Kit containing Dynole 31-2 (active drug) and 34-2 (negative control) (ab120474; Abcam, Cambridge, UK), $5 \mu \mathrm{M}$, were used to inhibit dynamin activity. Bafilomycin A1 (BafA1; Cell Signalling Technology), $10 \mathrm{nM}$, and ammonium chloride (NH4CL; 1145, Merck), $25 \mathrm{mM}$, were used to prevent $\mathrm{pH}$ acidification. The concentrations of the aforementioned inhibitors were used at the highest doses before affecting the cellular proliferation. Nystatin (Sigma-Aldrich), $10 \mu \mathrm{g} / \mathrm{mL}$, and methyl- $\beta$-cyclodextrin (M $\beta C D$; Sigma-Aldrich Chemie $\mathrm{GmbH}), 10 \mathrm{mM}$, were used to inhibit caveolae-mediated endocytosis. These concentrations were selected according to the literature [28,29] and also did not affect the proliferation of the cell lines used for the experiments.

Briefly, HeLa cells were seeded at a density of $8 \times 10^{4}$ cells/well in 24 -well plates and, on the following day, pre-treated for $45 \mathrm{~min}$ with the various inhibitors. Cells were then infected with recH-1PV-EGFP at a MOI of 0.2-0.3 transduction units (TU)/cell for $4 \mathrm{~h}$, washed twice with PBS and grown in culture medium for an additional $20 \mathrm{~h}$. At $24 \mathrm{~h}$ post-infection, cells were washed once with PBS, fixed with 3.7\% paraformaldehyde on ice for $15 \mathrm{~min}$, permeabilised with 1\% Triton X-100 for $10 \mathrm{~min}$ and stained with DAPI. Fluorescence images of EGFP-positive cells were acquired with a BZ-9000 fluorescence microscope (Keyence Corporation, Osaka, Japan) with 4X or 10X objective. DAPI staining was used to visualise the nuclei (cells). At least two independent experiments, each performed in duplicate, were performed for every condition tested.

\subsection{Cell Proliferation Assay}

Cell proliferation was monitored in real time through the xCELLigence system (ACEA Biosciences Inc., San Diego, CA, USA) according to the manufacturer's instructions. Briefly, $8 \times 10^{4} \mathrm{HeLa}$ or NCH125 cells per well were seeded in a 96-well E-plate (Roche) in a total volume of $100 \mu \mathrm{L}$ of complete DMEM medium. On the following day, cells were treated with different inhibitors for 45 min, and subsequently washed with PBS. Cell proliferation was monitored every $30 \mathrm{~min}$ in real time over a period of $72 \mathrm{~h}$. Data is expressed as "Normalised cell index", where all curves were normalised to an arbitrary value of 1.0 at the timepoint before treatment. Average values of each experimental condition assessed in triplicate are presented with the respective standard deviation (SD).

\section{6. siRNA-Mediated Knockdown}

Cells were seeded at a density of $4 \times 10^{4}$ cells/well in a 24-well plate and grown in $500 \mu \mathrm{L}$ of normal growth medium. After $24 \mathrm{~h}$, cells were transfected with $10 \mathrm{nM}$ siRNA using Lipofectamine RNAimax (Thermo Fisher Scientific, Carlsbad, CA, USA) according to the manufacturer's instructions. For AP2M1, we used the AP2M1 ON-TARGET plus Human siRNA SMARTpool (L-008170-00-0005) and, as a negative control, the plus Non-targeting pool (D-001810-10-05) (Dharmacon, Thermo Fisher Scientific). For CAV-1, we used two Silencer Select Validated siRNAs (s2446 and s2448; Life Technologies, Paisley, Scotland) and Silencer Select Negative Control \#2 siRNA (4390846, Life Technologies) as a control. After $24 \mathrm{~h}$, the medium was replaced, and cells were grown for an additional $24 \mathrm{~h}$ to allow efficient gene silencing. The cells were then infected for $24 \mathrm{~h}$ with recH-1PV-EGFP at 0.2-0.3 TU/cell. Cells were then washed once with PBS and processed as described above for fluorescence microscopy. At least two independent experiments, each performed in duplicate, were performed for every condition tested. 


\subsection{Western Blotting}

Cells were harvested, washed in PBS, and then lysed on ice for 30 min in RIPA buffer (10 mM Tris-HCl pH 7.5, $150 \mathrm{mM} \mathrm{NaCl}, 1 \mathrm{mM}$ EDTA pH 8, 1\% NP-40, 0.5\% Na-deoxylcholate and 0.5\% SDS supplemented with complete EDTA-free protease inhibitor (11697498001; Roche, Mannheim, Germany). Cellular debris was removed by centrifugation, and protein concentration in cell lysates was measured by bicinchoninic acid (BCA) assay (Thermo Fisher Scientific), according to manufacturer's instructions. SDS-PAGE analysis was performed on $50 \mu \mathrm{g}$ of total protein extract. After separation, proteins were transferred to Hybond-P membrane (GE Healthcare, Freiburg, Germany). Immunoblotting was carried out with the following antibodies: mouse monoclonal anti-vinculin (sc-25336; Santa Cruz Biotechnology, Heidelberg, Germany) and rabbit monoclonal CAV-1 (D46G3; Cell Signalling Technology) at 1:1000 dilution. After incubation with horseradish peroxidase conjugated secondary antibodies (Santa Cruz Biotechnology) at 1:1000 dilution, proteins were revealed with Western Blot Chemiluminescence Reagent Plus (Perkin Elmer Life Sciences) and exposed to Hyperfilm ${ }^{\mathrm{TM}}$ ECL radiographic films (GE Healthcare).

\section{Results}

\subsection{Electron Microscopy Analysis Reveals H-1PV within Clathrin-Coated Pits}

In order to investigate the H-1PV internalisation pathway, we performed EM analysis of HeLa cells infected with H-1PV. Infection was carried out at $4{ }^{\circ} \mathrm{C}$ for $1 \mathrm{~h}$ to allow virus cell surface binding. Cells were then shifted back to $37^{\circ} \mathrm{C}$ for various intervals to allow virus internalisation. EM analysis of infected cells at $4{ }^{\circ} \mathrm{C}$ showed the virus at the cell surface bound to thickened regions resembling clathrin-associated plasma membrane (Figure 1A) [30]. In the first $5 \mathrm{~min}$ after cells were shifted back to $37^{\circ} \mathrm{C}$, the invagination of clathrin-rich regions started with $\mathrm{H}-1 \mathrm{PV}$ particles remaining associated with these regions (Figure 1B). From 10 to $30 \mathrm{~min}$, viral particles were detected both in clathrin-coated pits (Figure 1C) or in the cytosol inside completely invaginated vesicles (we found up to nine particles in a single vesicle) (Figure 1D). Furthermore, in the course of the experiment, no virus internalisation was found in vesicles with the small flask-shaped invaginations that are characteristic of caveolae-mediated endocytosis [31], which suggests that H-1PV is internalised mainly (if not exclusively) via clathrin-mediated endocytosis.

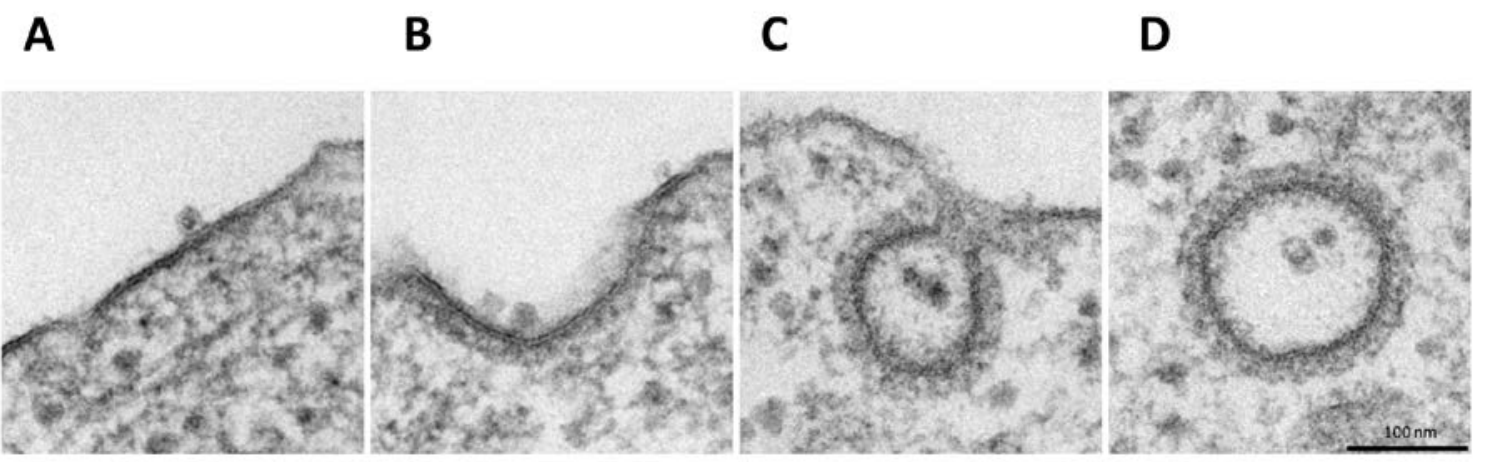

Figure 1. Endocytosis of H-1PV is clathrin-dependent. HeLa cells were infected with $\mathrm{H}-1 \mathrm{PV}$ for $1 \mathrm{~h}$ at $4{ }^{\circ} \mathrm{C}$ to allow H-1PV cell surface attachment but not entry. Cells were then shifted to $37^{\circ} \mathrm{C}$ to allow $\mathrm{H}-1 \mathrm{PV}$ cell internalisation. Cells were collected every $5 \mathrm{~min}$ for a total of $30 \mathrm{~min}$ and processed for EM analysis. (A) At $4{ }^{\circ} \mathrm{C}, \mathrm{H}-1 \mathrm{PV}$ particles are found attached to electro-dense (clathrin-rich) regions on the plasma membrane. (B) In the first 5 min after release at $37^{\circ} \mathrm{C}, \mathrm{H}-1 \mathrm{PV}$ particles are detected in early-forming clathrin-coated pits. (C) From 10 to $30 \mathrm{~min}, \mathrm{H}-1 \mathrm{PV}$ particles moved into the cells within deeply invaginated clathrin-coated pits that were still connected to the plasma membrane, forming an hourglass-like membrane neck. (D) Later in the infection (10-30 min at $\left.37^{\circ} \mathrm{C}\right), \mathrm{H}-1 \mathrm{PV}$ particles are seen being trafficked within the cell inside clathrin-coated vesicles. 


\subsection{H-1PV Co-Localises with Clathrin Upon Entry}

The EM analysis provided first evidence that H-1PV uses clathrin-mediated endocytosis to enter cells. To confirm these results independently, we checked for possible co-localisation of $\mathrm{H}-1 \mathrm{PV}$ and clathrin-heavy chain (CHC). To this end, HeLa cells were infected with H-1PV for $1 \mathrm{~h}$ at $4{ }^{\circ} \mathrm{C}$ and then shifted back to $37^{\circ} \mathrm{C}$. After $30 \mathrm{~min}$, a fraction of internalised H-1PV was clearly detected in association with $\mathrm{CHC}$ by confocal microscopy (Figure 2), providing further evidence that $\mathrm{H}-1 \mathrm{PV}$ is internalised through a clathrin-dependent pathway.
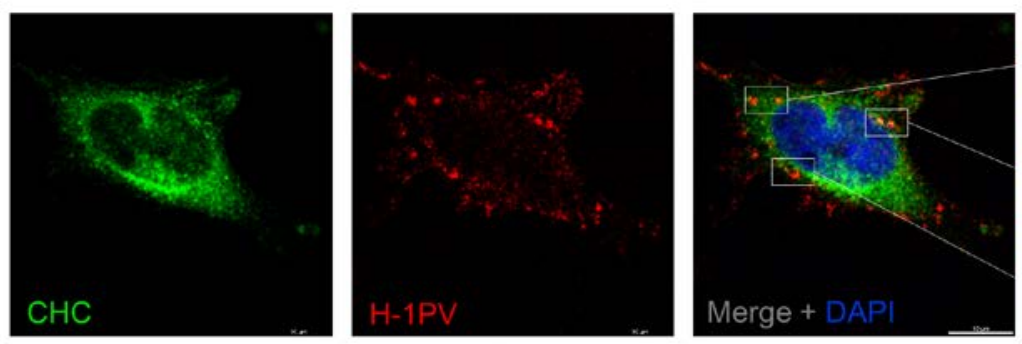

zoomed

Figure 2. H-1PV co-localises with clathrin upon entry. HeLa cells were infected with H-1PV for $1 \mathrm{~h}$ at $4{ }^{\circ} \mathrm{C}$ and then shifted to $37^{\circ} \mathrm{C}$ for $30 \mathrm{~min}$ before being processed for immunostaining using anti-H-1PV full capsid and anti-clathrin-heavy chain (CHC) antibodies. Cell nuclei were visualised by DAPI staining. Confocal microscopy analysis showed that H-1PV particles (Alexa Fluor 594, red) co-localised with CHC (Alexa Fluor 488, green) early upon infection. Three examples of regions where co-localisation is observed are framed by white boxes and shown zoomed in.

\subsection{H-1PV Enters Cells Preferentially via Clathrin-Mediated Endocytosis}

Next, we investigated whether targeting regulators of clathrin-mediated endocytosis would affect $\mathrm{H}-1 \mathrm{PV}$ infection. A recombinant H-1PV expressing the EGFP reporter gene (recH-1PV-EGFP) was used for the experiments. This non-replicative parvovirus shares the same capsid of the wild type but harbours the EGFP gene under the control of the natural P38 late promoter, whose activity is regulated by NS1 viral protein [25]. Therefore, the EGFP signal directly correlates to the ability of the virus to reach the nucleus and initiate its own gene transcription.

The effects of various inhibitors on H-1PV entry were assessed in HeLa and in glioma-derived NCH125 cell lines. The latter, like HeLa, is highly permissive to H-1PV infection. Pharmacological inhibitors included hypertonic sucrose, chlorpromazine (CPZ) and pitstop 2. Hypertonic sucrose is a classical inhibitor that traps clathrin in microcages [32]. CPZ is a cationic, amphiphilic drug that induces the misassembly of clathrin lattices at the cell surface and on endosomes [33]. Pitstop 2 interferes with the binding of proteins to the N-terminal domain of clathrin [34]. The internalisation of TexasRed-labelled transferrin, a protein known to be exclusively internalised through clathrin-mediated endocytosis, was monitored to check the effectiveness of each treatment [35]. At the concentrations used, the three inhibitors blocked transferrin uptake efficiently but did not affect cell proliferation (Supplementary Figure S1).

Pre-treatment with hypertonic sucrose decreased H-1PV transduction by more than $90 \%$ in HeLa and $80 \%$ in NCH125 cells compared to untreated cells (Figure 3A,B). When the compound was applied $3 \mathrm{~h}$ post-infection (by which time the virus is already internalised), no significant changes in H-1PV transduction were observed, indicating that the drug interferes with H-1PV transduction at the level of virus entry (Supplementary Figure S2). Strong inhibition of H-1PV transduction was also achieved by pre-treating cells with CPZ (approximately 90\% reduction in both cell lines) or with pitstop 2 (60\% reduction in Hela and over $70 \%$ in NCH125 cells) compared to untreated cells. 

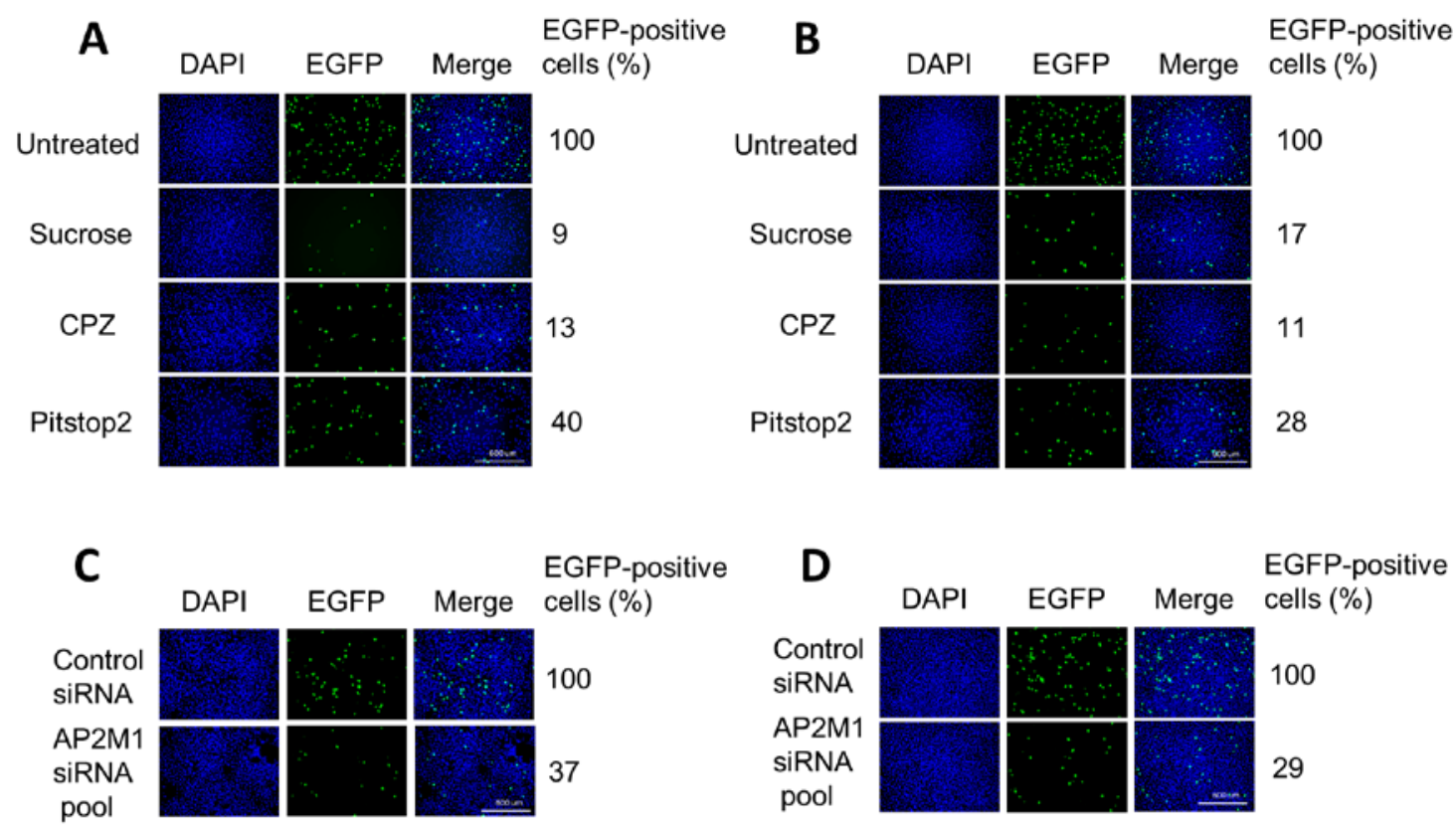

Figure 3. Blocking clathrin-mediated endocytosis results in a significant reduction of H-1PV transduction. (A) HeLa and (B) NCH125 cells were pre-treated with different clathrin-mediated endocytosis inhibitors (hypertonic sucrose, chlorpromazine (CPZ) or pitstop 2) or left untreated. Cells were then infected with recH-1PV-EGFP for $4 \mathrm{~h}$ in the presence of the inhibitor. At $20 \mathrm{~h}$ post-infection, cells were processed as described in the Materials and Methods (M\&M) section. (C) HeLa and (D) NCH125 cells were transfected with a pool of siRNAs targeting either AP2M1 or negative control. At $48 \mathrm{~h}$ post-transfection, cells were infected with recH-1PV-EGFP for $4 \mathrm{~h}$ and grown for an additional $20 \mathrm{~h}$. Cells were then processed as described in the M\&M section. Numbers represent the average percentage of EGFP-positive cells relative to the number of EGFP-positive cells observed in untreated or scrambled siRNA-transfected cells, which was arbitrarily set as $100 \%$.

The AP-2 complex is a heterotetramer that plays an essential role in clathrin-mediated endocytosis [21]. To confirm the involvement of clathrin-mediated endocytosis in H-1PV cell entry, we silenced the expression of AP2M1, the gene encoding subunit $\mu 1$ of AP-2 [36]. To this end, HeLa and NCH125 cells were transfected with either a siRNA pool targeting AP2M1 or scrambled siRNA (negative control), and subsequently infected with recH-1PV-EGFP. Under conditions in which the silencing of $A P 2 M 1$ successfully reduced transferrin uptake, we observed a strong decrease in H-1PV transduction (over 60\% compared to the scrambled siRNA-treated cells) in both cell lines (Figure 3C,D). Taken together, these results show that H-1PV uses clathrin-mediated endocytosis to enter HeLa and $\mathrm{NCH} 125$ cells.

\subsection{H-1PV Does Not Enter Cells via Caveolae-Dependent Endocytosis}

To investigate whether H-1PV uses pathways other than clathrin-mediated endocytosis to enter HeLa and NCH125 cancer cells, we inhibited clathrin-independent endocytosis using nystatin and methyl- $\beta$-cyclodextrin $(\mathrm{M} \beta \mathrm{CD}$ ). Both drugs selectively disrupt lipid rafts (e.g., cholesterol), including those required for caveolae-dependent entry $[28,29,37]$. Yet, neither nystatin nor $M \beta C D$ decreased $\mathrm{H}-1 \mathrm{PV}$ transduction, providing evidence that the virus does not use this endocytic route to enter these cells (Figure 4A,B). Similar results were also obtained using the two drugs at lower or higher concentrations $(0.1-100 \mu \mathrm{g} / \mathrm{mL}$ for nystatin, $0.1-100 \mu \mathrm{M}$ for $\mathrm{M} \beta \mathrm{CD})$. We also carried out siRNA-mediated knockdown of $C A V 1$ (which encodes for caveolin-1) by using two different siRNAs. Knock-down of CAV1 gene expression did not decrease H-1PV transduction activity compared with scrambled siRNA-transfected cells, but instead increased it (Figure 4C,D). Together, these results indicate that caveolae-dependent endocytosis is not involved in H-1PV entry of HeLa and NCH125 cells. 


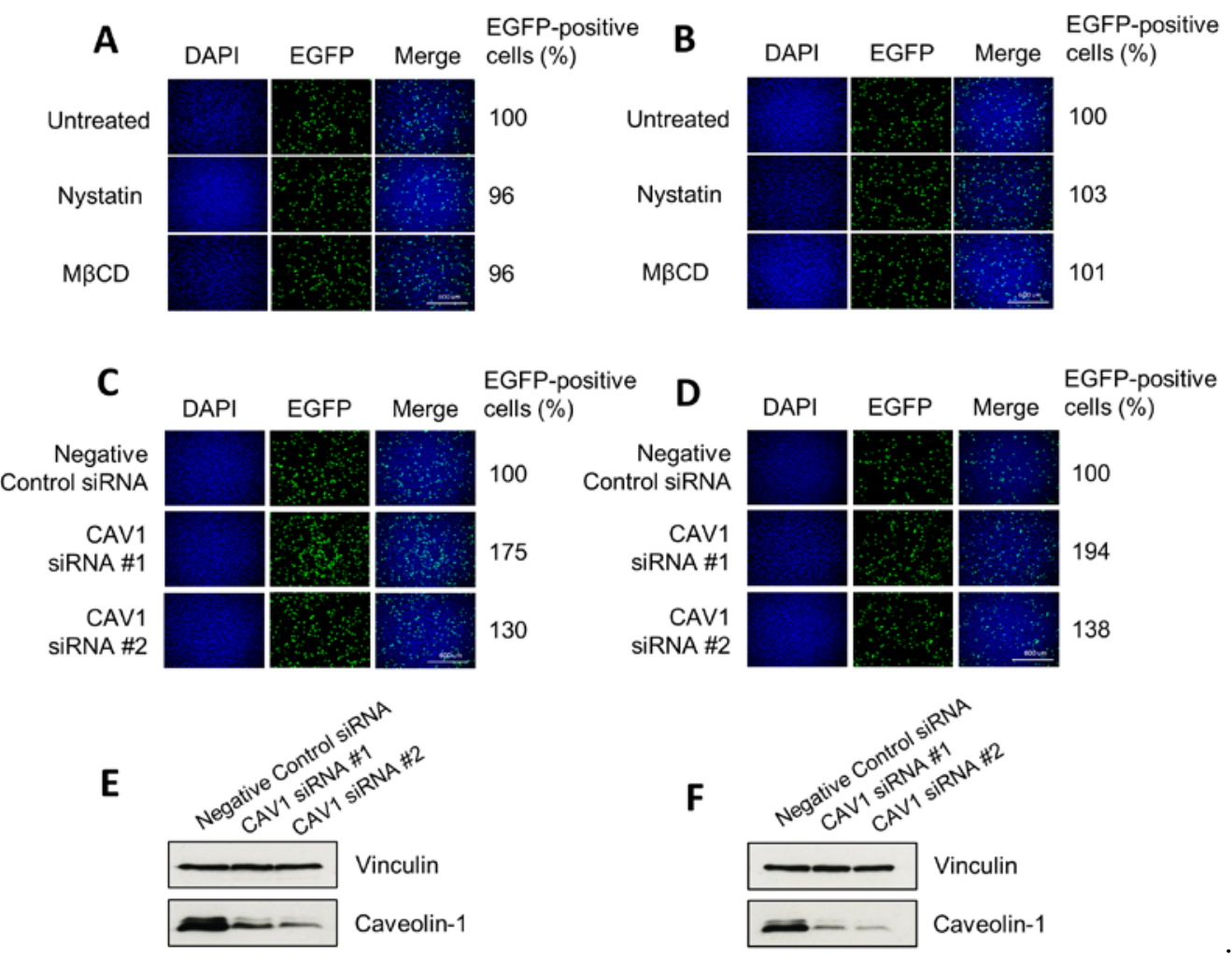

Figure 4. Disruption of clathrin-independent endocytosis does not decrease H-1PV transduction. (A) HeLa and (B) NCH125 cells were either pre-treated with cholesterol-sequestering drugs (nystatin or methyl- $\beta$ cyclodextrin $(M \beta C D)$ ) for $45 \mathrm{~min}$ or left untreated. Cells were then infected with recH-1PV-EGFP for $4 \mathrm{~h}$ in the presence (or absence) of the inhibitor. At $20 \mathrm{~h}$ post-infection, cells were processed as described in the M\&M section for immunofluorescence analysis. (C) HeLa and (D) NCH125 cells were transfected with siRNAs targeting CAV1 or a negative control siRNA. At $48 \mathrm{~h}$ post-transfection, cells were infected with recH-1PV-EGFP for $4 \mathrm{~h}$ and grown for an additional $20 \mathrm{~h}$. Cells were then processed as described in panel A. Numbers represent the average percentage of EGFP-positive cells relative to the number of EGFP-positive cells observed in untreated cells, which was arbitrarily set as $100 \%$. The steady protein levels of caveolin- 1 on lysates derived from (E) HeLa or (F) NCH125 siRNA-transfected cells were analysed by Western blotting. Vinculin was used as a loading control.

\subsection{H-1PV Internalisation Is Dependent on Dynamin}

Dynamin is a large GTPase with an essential role in cellular membrane fission for newly formed vesicles. It is therefore required for clathrin- and caveolae-mediated endocytosis but not for macropinocytosis [38]. We used the highly selective Dynole 34-2 to inhibit dynamin activity [39,40], and Dynole 31-2, its inactive form, as a negative control. At a concentration that blocked transferrin uptake, Dynole 34-2 drastically reduced virus transduction to just $8 \%$ in HeLa and $13 \%$ in NCH125 cells (Figure 5) compared to untreated cells. As expected, Dynole 31-2 did not have any significant effect on H-1PV transduction. These results demonstrate that dynamin plays an essential role in $\mathrm{H}-1 \mathrm{PV}$ infection. 


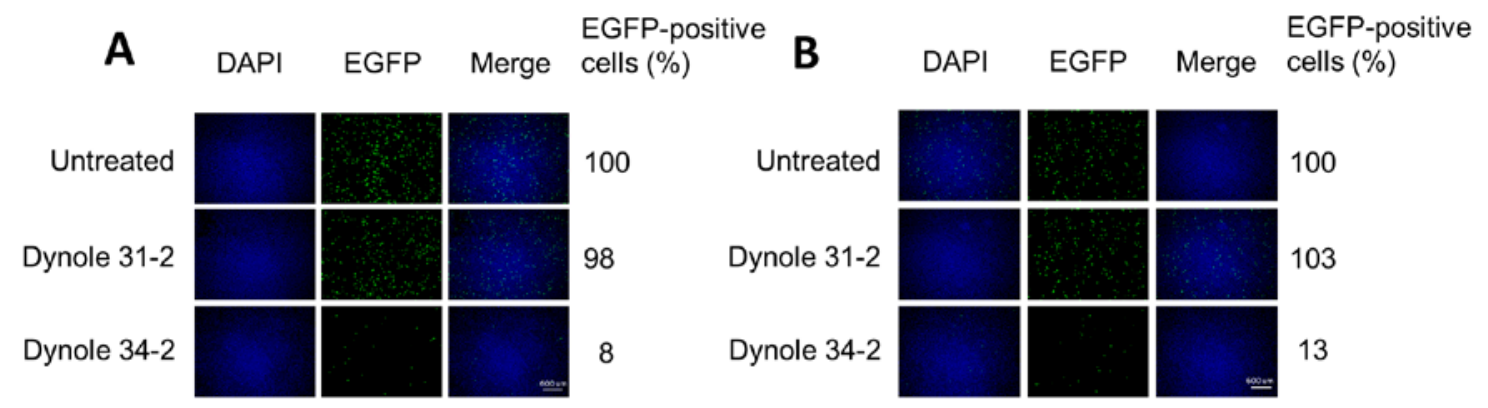

Figure 5. H-1PV requires dynamin activity for entry. (A) HeLa and (B) NCH125 cells were pre-treated with either Dynole 34-2 or its inactive form, Dynole 31-2. Cells were subsequently infected with recH-1PV-EGFP for $4 \mathrm{~h}$ in the presence of the inhibitor. At $20 \mathrm{~h}$ post-infection, cells were processed as described in Figure 4A. Numbers represent the average percentage of EGFP-positive cells relative to the number of EGFP-positive cells observed in untreated cells that was arbitrarily set as $100 \%$.

3.6. H-1PV Hijacks Endosomes for Trafficking into the Cytosol and Acidic $p H$ Is Required for Productive H-1PV Infection

The Rab family of proteins and their effectors play a key role in the formation, maintenance and trafficking of endosomes [41]. Early endosome antigen 1 (EEA1) is a Rab5 effector protein that is involved in sorting endocytic vesicles at the early endosome level [42]. Rab7 is considered to be the key regulator of late endosome trafficking. Lysosomal-associated membrane protein 1 (LAMP-1) is enriched in late endosomes and lysosomes, where, among other functions, it maintains lysosomal integrity and $\mathrm{pH}[43,44]$.

To provide direct evidence that H-1PV hijacks endocytosis for its intracellular trafficking, we infected HeLa cells with H-1PV for $1 \mathrm{~h}$, fixed the cells, and then stained viral capsids as well as EEA1, Rab7 and LAMP-1 proteins with antibodies. Confocal microscopy analysis showed that H-1PV co-localised with EEA1, Rab7 and LAMP-1 markers during infection (Figure 6A).

Next, we hypothesised that the acidic $\mathrm{pH}$ in the endocytic compartments would provide the environmental conditions required for a productive H-1PV infection [3] (as it does for other PtPV infections), possibly triggering the conformational changes necessary for uncoating and nuclear translocation. To test whether low endosomal $\mathrm{pH}$ is required for H-1PV infection, we pre-treated HeLa and $\mathrm{NCH} 125$ cells with ammonium chloride $\left(\mathrm{NH}_{4} \mathrm{Cl}\right)$, a lysosomotropic weak base [45], or bafilomycin A1 (BafA1), a blocker of vacuolar $\mathrm{H}^{+}$-ATPases [46]. Treatment with $\mathrm{NH}_{4} \mathrm{Cl}$ resulted in a strong decrease of virus transduction in both HeLa and NCH125 cells (39\% and 30\%, respectively) (Figure 6B,C), while treatment with BafA1 completely abolished H-1PV transduction (Figure 6D,E), in comparison to untreated cells. Together, these results provide evidence that H-1PV, like other PtPVs, requires acidic endosomal $\mathrm{pH}$ for a productive infection. 

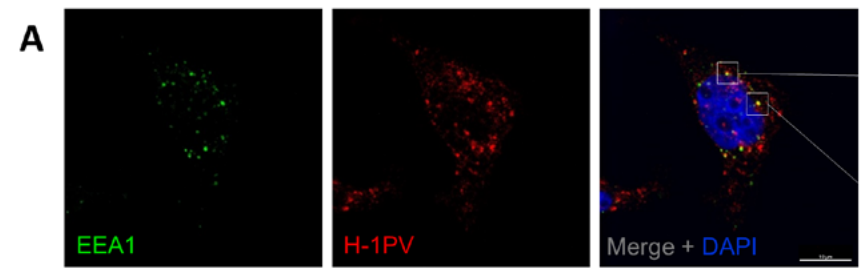

zoomed
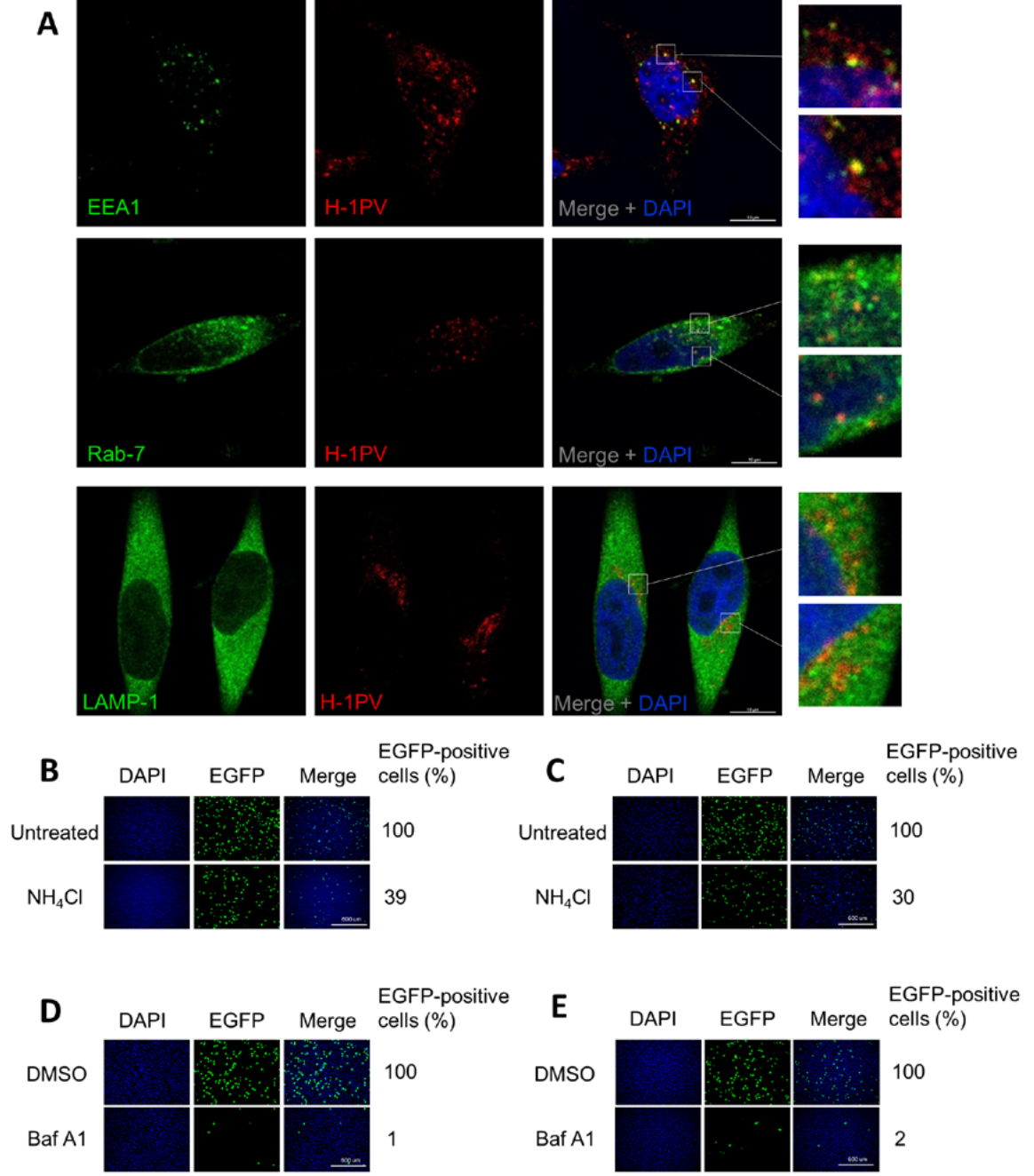

Figure 6. H-1PV trafficking occurs via the endosomal system with acidic $\mathrm{pH}$ being crucial for a productive infection. (A) HeLa cells were infected with $\mathrm{H}-1 \mathrm{PV}$ at MOI of $500 \mathrm{pfu} / \mathrm{cell}$ for $1 \mathrm{~h}$ at $4{ }^{\circ} \mathrm{C}$ and shifted to $37^{\circ} \mathrm{C}$ for $30 \mathrm{~min}$. Cells were then fixed, permeabilised and stained with DAPI and H-1PV capsid antibody, together with one of the endosomal markers (EEA1, Rab-7 or LAMP-1). Confocal microscopy analysis indicates that H-1PV particles (Alexa Fluor 594) co-localise with all three markers, EEA1, Rab-7 and LAMP-1 (Alexa Fluor 488), upon infection. Two examples of regions where co-localisation is observed are framed in white boxes and shown enlarged. (B,D) HeLa and (C,E) NCH125 cells were incubated with ammonium chloride $\left(\mathrm{NH}_{4} \mathrm{CL}\right)$ or bafilomycin $\mathrm{A} 1$ (BafA1) respectively, for $45 \mathrm{~min}$, or left untreated. Cells were subsequently infected with recH-1PV-EGFP for $4 \mathrm{~h}$ in the presence of the inhibitor. At $20 \mathrm{~h}$ post-infection, cells were processed as described in the M\&M section. Numbers represent the average percentage of EGFP-positive cells relative to the number of EGFP-positive cells observed in untreated cells, which was arbitrarily set as $100 \%$.

\section{Discussion}

$\mathrm{H}-1 \mathrm{PV}$ is a promising oncolytic virus. Early-phase clinical studies show that virus treatment is safe, well-tolerated and associated with first signs of anticancer efficacy. The present study aimed to fill a gap in knowledge of H-1PV biology regarding the pathways used by this virus to enter cancer cells. We chose two cancer cell lines as models to investigate the early steps of H-1PV infection: the cervical carcinoma-derived HeLa cell line and the glioma-derived NCH125 cell line. Previous laboratory studies have shown that these two cell lines are highly permissive to H-1PV infection and susceptible to its oncolytic activity $[7,24,47]$. 
In our study, for the first time, we show that H-1PV exploits clathrin-mediated endocytosis to enter cancer cells. EM analysis of H-1PV-infected HeLa cells revealed virus particles associated with clathrin-coated pits and then internalised inside clathrin-coated vesicles (Figure 1). In agreement with this finding, confocal microscopy analysis showed co-localisation of H-1PV and clathrin (Figure 2). Furthermore, pharmacological inhibition of clathrin-mediated endocytosis using hypertonic sucrose, CPZ and pitstop 2, as well as siRNA-mediated silencing of AP2M1, a key regulator of clathrin-mediated endocytosis, confirmed the heavy dependence of $\mathrm{H}-1 \mathrm{PV}$ on this pathway for successful entry into both HeLa and NCH125 cancer cells (Figure 3).

Previous research has reported that other PtPVs use clathrin-mediated endocytosis to gain access to cells and progress the infection (reviewed in Reference [3]). Our results are therefore in agreement with the idea that clathrin-mediated endocytosis is the default entry pathway for PtPVs. However, a number of PtPVs have also been shown to hijack alternative endocytic pathways. For instance, MVMp uses at least three different endocytic routes, such as clathrin-, caveolae- and clathrin-independent carrier-mediated endocytosis [22]. Moreover, PPV uses both clathrin-dependent endocytosis and macropinocytosis [16]. FPV and CPV are taken up by cells via binding to the transferrin receptor, which is typically endocytosed by clathrin-mediated endocytosis. However, FPV may also enter cells through alternative internalisation mechanisms, as deletions or mutations in the internalisation motif of the transferrin receptor, while decreasing FPV cellular uptake, did not completely arrest viral infection [48]. Although we cannot completely rule out the possibility that $\mathrm{H}-1 \mathrm{PV}$, like other PtPVs, can also use different pathways to enter HeLa and NCH125 cancer cells, our results seem to exclude caveolae-mediated endocytosis as a major entry pathway. Indeed, pre-treatment of cells with nystatin and $\mathrm{M} \beta \mathrm{CD}$, two inhibitors of caveolae-mediated endocytosis, did not decrease H-1PV transduction levels, suggesting that these drugs did not modify H-1PV entry. These results are in agreement with previous studies showing that bovine parvovirus (BPV) [17] and PPV [16] do not use caveolae-mediated endocytosis for their cell internalisation. Surprisingly, CAV1 siRNA-mediated knockdown increased H-1PV transduction (Figure 4), suggesting that caveolin-1 (the protein encoded by $C A V 1$ ) interferes with $\mathrm{H}-1 \mathrm{PV}$ infection. Human immunodeficiency virus (HIV) infection is restricted by caveolin- 1 in various ways, e.g., at the transcriptional level by suppressing NF-kB p65 acetylation in macrophages, or by interacting with HIV viral proteins to impair viral infectivity [49,50]. Similarly, abundant caveolin-1 levels prevent Influenza A virus from infecting mouse embryo fibroblasts, which is reversed by depleting caveolin-1 [51]. Further experiments are required to find out whether and at what level caveolin-1 represents a negative modulator of H-1PV infection. Along these lines, caveolin-1 antagonists or inhibitors could offer an interesting strategy to improve H-1PV efficacy in cancer with elevated levels of caveolin-1.

Our study also provides important evidence that dynamin is involved in H-1PV entry (Figure 5). Dynamin is also required for MVMp entry in murine A9 fibroblasts, a process that occurs through both clathrin- and caveolae-mediated endocytosis [22]. However, the same study showed that dynamin is not involved in MVMp entry into mouse mammary cells transformed with polyomavirus middle $\mathrm{T}$ antigen, which instead occurs via clathrin-independent carrier-mediated endocytosis [22].

Another cell entry route used by viruses is macropinocytosis, generally described as a dynamin-independent process [52,53]. Among PtPVs, PPV has been reported to use this route of entry [16]. However, we showed that inhibition of dynamin almost completely abolished H-1PV infection, which makes it unlikely that H-1PV uses macropinocytosis as an alternative pathway to enter HeLa and NCH125 cancer cells.

Numerous viruses are known to hijack Rab-dependent pathways to enter cells. Of these, Rab5 and Rab7 GTPases are the key regulators of transport to early and late endosomes, while LAMP-1 is present mainly in late endosomes/lysosomes [41,54]. In the present study, we show that H-1PV particles co-localise with EEA1, an early endosome marker, and with Rab7 and LAMP-1, two late endosome markers (Figure 6A), indicating that H-1PV, like other PtPVs [3], uses endosomes for its cytosolic trafficking into the cells. However, a fraction of H-1PV particles is most likely trapped 
in LAMP1-positive lysosomes. This has been observed for other PtPVs such as MVM, for which sequestration in LAMP1-positive lysosomes limits the efficiency of its nuclear translocation [55], and CPV, which accumulates in perinuclear LAMP2-positive lysosomes [56].

Previous studies have shown that the acidic environment inside the endosomes changes in redox conditions, and acid proteases and phosphatases drive the conformational rearrangements in the catalytic phospholipase 2 domain of the VP1 protein $[55,57,58]$. These changes are required first for the release of PtPV particles from the late endosome to the cytosol, and then for translocation into the nucleus $[23,57,59,60]$. For instance, the endosomal acidic environment is required for a productive infection of B19V [20], CPV [18,61,62] and MVM [55,63], among other parvoviruses. In agreement with these observations, we also found that $\mathrm{NH}_{4} \mathrm{Cl}$ and BafA1 strongly hampered $\mathrm{H}-1 \mathrm{PV}$ transduction (Figure 6).

In summary, our study shows, for the first time, that H-1PV internalisation occurs via clathrin-mediated and dynamin-dependent endocytosis, while requiring endosomal acidification, with EEA1 and Rab7 involved in the infection process. However, we cannot rule out the possibility of $\mathrm{H}-1 \mathrm{PV}$ taking other pathways in other cell types. Important questions remain, namely which receptor $\mathrm{H}-1 \mathrm{PV}$ uses to initiate uptake and whether other co-receptors are involved. In addition, the exact mechanisms of endosomal escape and subsequent nuclear entry, and finally the site where the H-1PV genome becomes accessible for replication, need to be investigated [3]. A better understanding of the early steps of H-1PV (and, more generally, PtPV) infection is crucial not only to decipher viral tropism and inherent oncolytic properties, but also to improve the clinical potential of H-1PV in cancer virotherapy.

Supplementary Materials: The following are available online at http://www.mdpi.com/1999-4915/12/10/1199/s1, Figure S1: Entry pathway inhibitors did not alter cell proliferation. Figure S2: Sucrose, a classical inhibitor of clathrin-mediated endocytosis, has no impact on virus transduction if added $4 \mathrm{~h}$ after virus infection.

Author Contributions: Conceptualisation, T.F. and A.M.; methodologies, T.F., A.K., M.E. and K.R.; investigation, T.F., C.B. (confocal microscopy analysis) and K.R. (EM analysis); data curation, T.F. and A.M.; writing-original draft preparation, T.F. and A.M.; writing-review and editing, A.M.; visualisation, T.F., C.B. and A.M.; supervision, A.M.; funding acquisition, A.M. All authors have read and agreed to the published version of the manuscript.

Funding: This study has been partially funded by a research grant from the Cooperational Research Program of the Deutsches Krebsforschungszentrum, Heidelberg, and the Ministry of Science and Technology (MOST), Israel, to A.M. and M.E.

Acknowledgments: We are grateful to Barbara Leuchs (DKFZ, Heidelberg, Germany) for kindly providing the H-1PV capsid monoclonal antibody. We would also like to extend our acknowledgements to Tiina Marttila for producing both the H-1PV wild-type and the recH-1PV-EGFP viruses. We thank Caroline Hadley (INLEXIO) for critically reading the manuscript.

Conflicts of Interest: A.M. is co-inventor in several H-1PV-related patents. No other conflicts of interest are declared by the authors.

\section{References}

1. Cotmore, S.F.; Tattersall, P. Parvoviral host range and cell entry mechanisms. Adv. Virus Res. 2007, 70, $183-232$. [PubMed]

2. Cotmore, S.F.; Agbandje-McKenna, M.; Chiorini, J.A.; Mukha, D.V.; Pintel, D.J.; Qiu, J.; Soderlund-Venermo, M.; Tattersall, P.; Tijssen, P.; Gatherer, D. The family parvoviridae. Arch. Virol. 2014, 159, 1239-1247. [CrossRef] [PubMed]

3. Ros, C.; Bayat, N.; Wolfisberg, R.; Almendral, J.M.J.V. Protoparvovirus cell entry. Viruses 2017, 9, 313. [CrossRef] [PubMed]

4. Bretscher, C.; Marchini, A. H-1 parvovirus as a cancer-killing agent: Past, present, and future. Viruses 2019, 11, 562. [CrossRef] [PubMed]

5. Hartley, A.; Kavishwar, G.; Salvato, I.; Marchini, A. A roadmap for the success of oncolytic parvovirus-based anticancer therapies. Annu. Rev. Virol. 2020, 7, 537-557. [CrossRef] 
6. Marchini, A.; Daeffler, L.; Pozdeev, V.I.; Angelova, A.; Rommelaere, J. Immune conversion of tumor microenvironment by oncolytic viruses: The protoparvovirus H-1PV case study. Front. Immunol. 2019, 10, 1848. [CrossRef]

7. Hristov, G.; Kramer, M.; Li, J.; El-Andaloussi, N.; Mora, R.; Daeffler, L.; Zentgraf, H.; Rommelaere, J.; Marchini, A. through its nonstructural protein NS1, parvovirus H-1 induces apoptosis via accumulation of reactive oxygen species. J. Virol. 2010, 84, 5909-5922. [CrossRef]

8. Geletneky, K.; Huesing, J.; Rommelaere, J.; Schlehofer, J.R.; Leuchs, B.; Dahm, M.; Krebs, O.; von Knebel Doeberitz, M.; Huber, B.; Hajda, J. Phase I/IIa study of intratumoral/intracerebral or intravenous/intracerebral administration of Parvovirus H-1 (ParvOryx) in patients with progressive primary or recurrent glioblastoma multiforme: ParvOryx01 protocol. BMC Cancer 2012, 12, 99. [CrossRef]

9. Hajda, J.; Lehmann, M.; Krebs, O.; Kieser, M.; Geletneky, K.; Jäger, D.; Dahm, M.; Huber, B.; Schöning, T.; Sedlaczek, O. A non-controlled, single arm, open label, phase II study of intravenous and intratumoral administration of ParvOryx in patients with metastatic, inoperable pancreatic cancer: ParvOryx02 protocol. BMC Cancer 2017, 17, 1-11. [CrossRef]

10. Nüesch, J.P.; Lacroix, J.; Marchini, A.; Rommelaere, J. Molecular pathways: Rodent parvovirusesMechanisms of oncolysis and prospects for clinical cancer treatment. Clin. Cancer Res. 2012, 18, 3516-3523. [CrossRef]

11. López-Bueno, A.; Rubio, M.-P.; Bryant, N.; McKenna, R.; Agbandje-McKenna, M.; Almendral, J.M. Host-selected amino acid changes at the sialic acid binding pocket of the parvovirus capsid modulate cell binding affinity and determine virulence. J. Virol. 2006, 80, 1563-1573. [CrossRef] [PubMed]

12. Allaume, X.; El-Andaloussi, N.; Leuchs, B.; Bonifati, S.; Kulkarni, A.; Marttila, T.; Kaufmann, J.K.; Nettelbeck, D.M.; Kleinschmidt, J.; Rommelaere, J. Retargeting of rat parvovirus H-1PV to cancer cells through genetic engineering of the viral capsid. J. Virol. 2012, 86, 3452-3465. [CrossRef] [PubMed]

13. Harbison, C.E.; Chiorini, J.A.; Parrish, C.R. The parvovirus capsid odyssey: From the cell surface to the nucleus. Trends Microbiol. 2008, 16, 208-214. [CrossRef]

14. Doherty, G.J.; McMahon, H.T. Mechanisms of endocytosis. Annu. Rev. Biochem. 2009, 78, 857-902. [CrossRef] [PubMed]

15. Mercer, J.; Schelhaas, M.; Helenius, A. Virus entry by endocytosis. Annu. Rev. Biochem. 2010, 79, $803-833$. [CrossRef]

16. Boisvert, M.; Fernandes, S.; Tijssen, P. Multiple pathways involved in porcine parvovirus cellular entry and trafficking toward the nucleus. J. Virol. 2010, 84, 7782-7792. [CrossRef]

17. Dudleenamjil, E.; Lin, C.-Y.; Dredge, D.; Murray, B.K.; Robison, R.A.; Johnson, F.B. Bovine parvovirus uses clathrin-mediated endocytosis for cell entry. J. Gen. Virol. 2010, 91, 3032-3041. [CrossRef]

18. Parker, J.S.; Parrish, C.R. Cellular uptake and infection by canine parvovirus involves rapid dynamin-regulated clathrin-mediated endocytosis, followed by slower intracellular trafficking. J. Virol. 2000, 74, 1919-1930. [CrossRef]

19. Vendeville, A.; Ravallec, M.; Jousset, F.-X.; Devise, M.; Mutuel, D.; López-Ferber, M.; Fournier, P.; Dupressoir, T.; Ogliastro, M. Densovirus infectious pathway requires clathrin-mediated endocytosis followed by trafficking to the nucleus. J. Virol. 2009, 83, 4678-4689. [CrossRef]

20. Quattrocchi, S.; Ruprecht, N.; Bönsch, C.; Bieli, S.; Zürcher, C.; Boller, K.; Kempf, C.; Ros, C. Characterization of the early steps of human parvovirus B19 infection. J. Virol. 2012, 86, 9274-9284. [CrossRef]

21. McMahon, H.T.; Boucrot, E. Molecular mechanism and physiological functions of clathrin-mediated endocytosis. Nat. Rev. Mol. Cell Biol. 2011, 12, 517. [CrossRef] [PubMed]

22. Garcin, P.O.; Panté, N. The minute virus of mice exploits different endocytic pathways for cellular uptake. Virology 2015, 482, 157-166. [CrossRef] [PubMed]

23. Suikkanen, S.; Aaltonen, T.; Nevalainen, M.; Välilehto, O.; Lindholm, L.; Vuento, M.; Vihinen-Ranta, M. Exploitation of microtubule cytoskeleton and dynein during parvoviral traffic toward the nucleus. J. Virol. 2003, 77, 10270-10279. [CrossRef] [PubMed]

24. Di Piazza, M.; Mader, C.; Geletneky, K.; y Calle, M.H.; Weber, E.; Schlehofer, J.; Deleu, L.; Rommelaere, J. Cytosolic activation of cathepsins mediates parvovirus H-1-induced killing of cisplatin and TRAIL-resistant glioma cells. J. Virol. 2007, 81, 4186-4198. [CrossRef] 
25. El-Andaloussi, N.; Endele, M.; Leuchs, B.; Bonifati, S.; Kleinschmidt, J.; Rommelaere, J.; Marchini, A. Novel adenovirus-based helper system to support production of recombinant parvovirus. Cancer Gene Ther. 2011, 18, 240-249. [CrossRef]

26. El-Andaloussi, N.; Leuchs, B.; Bonifati, S.; Rommelaere, J.; Marchini, A. Efficient recombinant parvovirus production with the help of adenovirus-derived systems. J. Vis. Exp. 2012, 62, e3518. [CrossRef]

27. Leuchs, B.; Roscher, M.; Muller, M.; Kurschner, K.; Rommelaere, J. Standardized large-scale H-1PV production process with efficient quality and quantity monitoring. J. Virol. Methods 2016, 229, 48-59. [CrossRef]

28. Rodal, S.K.; Skretting, G.; Garred, Ø.; Vilhardt, F.; Van Deurs, B.; Sandvig, K. Extraction of cholesterol with methyl- $\beta$-cyclodextrin perturbs formation of clathrin-coated endocytic vesicles. Mol. Biol. Cell 1999, 10, 961-974. [CrossRef]

29. Anderson, H.; Chen, Y.; Norkin, L. Bound simian virus 40 translocates to caveolin-enriched membrane domains, and its entry is inhibited by drugs that selectively disrupt caveolae. Mol. Biol. Cell 1996, 7, 1825-1834. [CrossRef]

30. Locker, J.K.; Schmid, S.L. Integrated electron microscopy: Super-duper resolution. PLoS Biol. 2013, 11, e1001639.

31. Short, B. A cell-free screen of caveolae interactions. J. Cell Biol. 2018, 217, 1883. [CrossRef]

32. Heuser, J.E.; Anderson, R. Hypertonic media inhibit receptor-mediated endocytosis by blocking clathrin-coated pit formation. J. Cell Biol. 1989, 108, 389-400. [CrossRef]

33. Wang, L.-H.; Rothberg, K.G.; Anderson, R. Mis-assembly of clathrin lattices on endosomes reveals a regulatory switch for coated pit formation. J. Cell Biol. 1993, 123, 1107-1117. [CrossRef]

34. Von Kleist, L.; Stahlschmidt, W.; Bulut, H.; Gromova, K.; Puchkov, D.; Robertson, M.J.; MacGregor, K.A.; Tomilin, N.; Pechstein, A.; Chau, N. Role of the clathrin terminal domain in regulating coated pit dynamics revealed by small molecule inhibition. Cell 2011, 146, 471-484. [CrossRef] [PubMed]

35. Mayle, K.M.; Le, A.M.; Kamei, D.T. The intracellular trafficking pathway of transferrin. Biochim. Biophys. Acta 2012, 1820, 264-281. [CrossRef] [PubMed]

36. Kadlecova, Z.; Spielman, S.J.; Loerke, D.; Mohanakrishnan, A.; Reed, D.K.; Schmid, S.L. Regulation of clathrin-mediated endocytosis by hierarchical allosteric activation of AP2. J. Cell Biol. 2017, 216, 167-179. [CrossRef] [PubMed]

37. Kilsdonk, E.P.; Yancey, P.G.; Stoudt, G.W.; Bangerter, F.W.; Johnson, W.J.; Phillips, M.C.; Rothblat, G.H. Cellular cholesterol efflux mediated by cyclodextrins. J. Biol. Chem. 1995, 270, 17250-17256. [CrossRef] [PubMed]

38. Singh, M.; Jadhav, H.R.; Bhatt, T. Dynamin functions and ligands: Classical mechanisms behind. Mol. Pharmacol. 2017, 91, 123-134. [CrossRef]

39. Hill, T.A.; Gordon, C.P.; McGeachie, A.B.; Venn-Brown, B.; Odell, L.R.; Chau, N.; Quan, A.; Mariana, A.; Sakoff, J.A.; Chircop, M. Inhibition of dynamin mediated endocytosis by the Dynoles-Synthesis and functional activity of a family of indoles. J. Med. Chem. 2009, 52, 3762-3773. [CrossRef]

40. Robertson, M.J.; Deane, F.M.; Robinson, P.J.; McCluskey, A. Synthesis of Dynole 34-2, Dynole 2-24 and Dyngo 4a for investigating dynamin GTPase. Nat. Protoc. 2014, 9, 851-870. [CrossRef]

41. Jordens, I.; Marsman, M.; Kuijl, C.; Neefjes, J. Rab proteins, connecting transport and vesicle fusion. Traffic 2005, 6, 1070-1077. [CrossRef] [PubMed]

42. Wilson, J.M.; De Hoop, M.; Zorzi, N.; Toh, B.-H.; Dotti, C.G.; Parton, R.G. EEA1, a tethering protein of the early sorting endosome, shows a polarized distribution in hippocampal neurons, epithelial cells, and fibroblasts. Mol. Biol. Cell 2000, 11, 2657-2671. [CrossRef] [PubMed]

43. Eskelinen, E.-L.; Tanaka, Y.; Saftig, P. At the acidic edge: Emerging functions for lysosomal membrane proteins. Trends Cell Biol. 2003, 13, 137-145. [CrossRef]

44. Eskelinen, E.-L. Roles of LAMP-1 and LAMP-2 in lysosome biogenesis and autophagy. Mol. Asp. Med. 2006, 27, 495-502. [CrossRef]

45. Misinzo, G.; Delputte, P.L.; Nauwynck, H.J. Inhibition of endosome-lysosome system acidification enhances porcine circovirus 2 infection of porcine epithelial cells. J. Virol. 2008, 82, 1128-1135. [CrossRef]

46. Yoshimori, T.; Yamamoto, A.; Moriyama, Y.; Futai, M.; Tashiro, Y. Bafilomycin A1, a specific inhibitor of vacuolar-type $\left.\mathrm{H}^{+}\right)$-ATPase, inhibits acidification and protein degradation in lysosomes of cultured cells. J. Biol. Chem. 1991, 266, 17707-17712. 
47. Li, J.; Bonifati, S.; Hristov, G.; Marttila, T.; Valmary-Degano, S.; Stanzel, S.; Schnolzer, M.; Mougin, C.; Aprahamian, M.; Grekova, S.P.; et al. Synergistic combination of valproic acid and oncolytic parvovirus $\mathrm{H}-1 \mathrm{PV}$ as a potential therapy against cervical and pancreatic carcinomas. EMBO Mol. Med. 2013, 5, 1537-1555. [CrossRef]

48. Hueffer, K.; Palermo, L.M.; Parrish, C.R. Parvovirus infection of cells by using variants of the feline transferrin receptor altering clathrin-mediated endocytosis, membrane domain localization, and capsid-binding domains. J. Virol. 2004, 78, 5601-5611. [CrossRef]

49. Simmons, G.E., Jr.; Taylor, H.E.; Hildreth, J.E. Caveolin-1 suppresses Human Immunodeficiency virus-1 replication by inhibiting acetylation of NF-кB. Virology 2012, 432, 110-119. [CrossRef]

50. Lin, S.; Nadeau, P.E.; Wang, X.; Mergia, A. Caveolin-1 reduces HIV-1 infectivity by restoration of HIV Nef mediated impairment of cholesterol efflux by apoA-I. Retrovirology 2012, 9, 1-16. [CrossRef]

51. Bohm, K.; Sun, L.; Thakor, D.; Wirth, M. Caveolin-1 limits human influenza A virus (H1N1) propagation in mouse embryo-derived fibroblasts. Virology 2014, 462, 241-253. [CrossRef] [PubMed]

52. Swanson, J.A.; Watts, C. Macropinocytosis. Trends Cell Biol. 1995, 5, 424-428. [CrossRef]

53. Preta, G.; Cronin, J.G.; Sheldon, I.M. Dynasore-not just a dynamin inhibitor. Cell Commun. Signal. 2015, 13, 24. [CrossRef]

54. Zhang, Y.-N.; Liu, Y.-Y.; Xiao, F.-C.; Liu, C.-C.; Liang, X.-D.; Chen, J.; Zhou, J.; Baloch, A.S.; Kan, L.; Zhou, B. Rab5, Rab7, and Rab11 are required for Caveola-dependent endocytosis of classical swine fever virus in porcine alveolar macrophages. J. Virol. 2018, 92, e00797-18. [CrossRef] [PubMed]

55. Mani, B.; Baltzer, C.; Valle, N.; Almendral, J.M.; Kempf, C.; Ros, C. Low pH-dependent endosomal processing of the incoming parvovirus minute virus of mice virion leads to externalization of the VP1 N-terminal sequence (N-VP1), N-VP2 cleavage, and uncoating of the full-length genome. J. Virol. 2006, 80, 1015-1024. [CrossRef]

56. Suikkanen, S.; Sääjärvi, K.; Hirsimäki, J.; Välilehto, O.; Reunanen, H.; Vihinen-Ranta, M.; Vuento, M. Role of recycling endosomes and lysosomes in dynein-dependent entry of canine parvovirus. J. Virol. 2002, 76, 4401-4411. [CrossRef]

57. Zádori, Z.; Szelei, J.; Lacoste, M.-C.; Li, Y.; Gariépy, S.; Raymond, P.; Allaire, M.; Nabi, I.R.; Tijssen, P. A viral phospholipase A2 is required for parvovirus infectivity. Dev. Cell 2001, 1, 291-302. [CrossRef]

58. Farr, G.A.; Zhang, L.-g.; Tattersall, P. Parvoviral virions deploy a capsid-tethered lipolytic enzyme to breach the endosomal membrane during cell entry. Proc. Natl. Acad. Sci. USA 2005, 102, 17148-17153. [CrossRef]

59. Canaan, S.; Zádori, Z.; Ghomashchi, F.; Bollinger, J.; Sadilek, M.; Moreau, M.E.; Tijssen, P.; Gelb, M.H. Interfacial enzymology of parvovirus phospholipases A2. J. Biol. Chem. 2004, 279, 14502-14508. [CrossRef] [PubMed]

60. Vihinen-Ranta, M.; Wang, D.; Weichert, W.S.; Parrish, C.R. The VP1 N-terminal sequence of canine parvovirus affects nuclear transport of capsids and efficient cell infection. J. Virol. 2002, 76, 1884-1891. [CrossRef] [PubMed]

61. Vihinen-Ranta, M.; Kalela, A.; Mäkinen, P.; Kakkola, L.; Marjomäki, V.; Vuento, M. Intracellular route of canine parvovirus entry. J. Virol. 1998, 72, 802-806. [CrossRef] [PubMed]

62. Basak, S.; Turner, H. Infectious entry pathway for canine parvovirus. Virology 1992, 186, 368-376. [CrossRef]

63. Ros, C.; Burckhardt, C.J.; Kempf, C. Cytoplasmic trafficking of minute virus of mice: Low-pH requirement, routing to late endosomes, and proteasome interaction. J. Virol. 2002, 76, 12634-12645. [CrossRef] [PubMed]

Publisher's Note: MDPI stays neutral with regard to jurisdictional claims in published maps and institutional affiliations.

(C) 2020 by the authors. Licensee MDPI, Basel, Switzerland. This article is an open access article distributed under the terms and conditions of the Creative Commons Attribution (CC BY) license (http://creativecommons.org/licenses/by/4.0/). 